\title{
Home Security Using Arduino
}

\author{
Kirtik Ayush \\ (3 ${ }^{\text {rd }}$ Year B. Tech) \\ Department of Electronics and Instrumentation Engineering (EIE), \\ Techno India Main College, Salt Lake, Kolkata, West Bengal, Kolkata:700159, India.
}

\begin{abstract}
:
This paper is intended to design and construct a simple home security device by using Arduino. The home security has recently become one of the most vital topics of the industry due to an increase in crime rate and also with the advancement of the technology.Arduino based RFID system alongside the numeric combination can be very secure and hassle free.
\end{abstract}

Keywords: RFID, EM-18, Arduino Uno,REES52, Buzzer, Home security

\section{Introduction}

Home security has recently become one of the most vital topics of the industry due to an increase in crime rate and also with the advancement of the technology. Padlock always has the chance of getting picked and broken through if done correctly. The immediate solution coming to the mind is identity based locks which require confirmation of the user trying to access the lock. RFID tag is one of the many solutions that can be implemented to ensure that only the person given access to the area can unlock the door. RFID tags are also cheap and easy to use compared to the traditional lock and key method. Adding to this is a number combination lock. This ensures that even if one of the security method is breached, the other one can prevent the unauthorized personnel to enter.

\section{Objective}

* To construct a simple home security device by using Arduino

* To design two layer protection using Arduino for home security

\section{Components Used}

* RFID Tags: RFID or Radio-Frequency Identification as its name suggests uses radio waves to communicate with the RFID reader. Its used in many places today like in employee badges. Using a passive RFID tag, it could be more cost efficient. Passive tag means that it will need to be powered by the reader when coming in contact to transmit data.

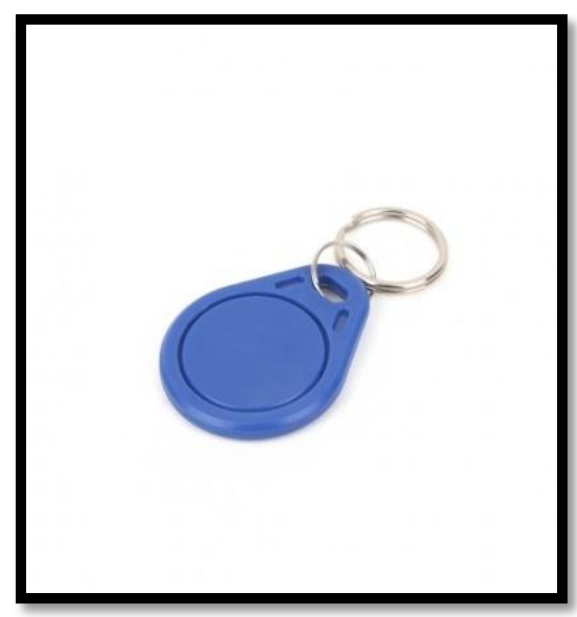

Source: https://www.mikroe.com/rfid-tag-1356mhz

* RFID Reader: The conversion of the radio waves from the tags to data is done by the RFID reader. It converts it then sends the data to main computer for the data to be processed and action to be taken accordingly.

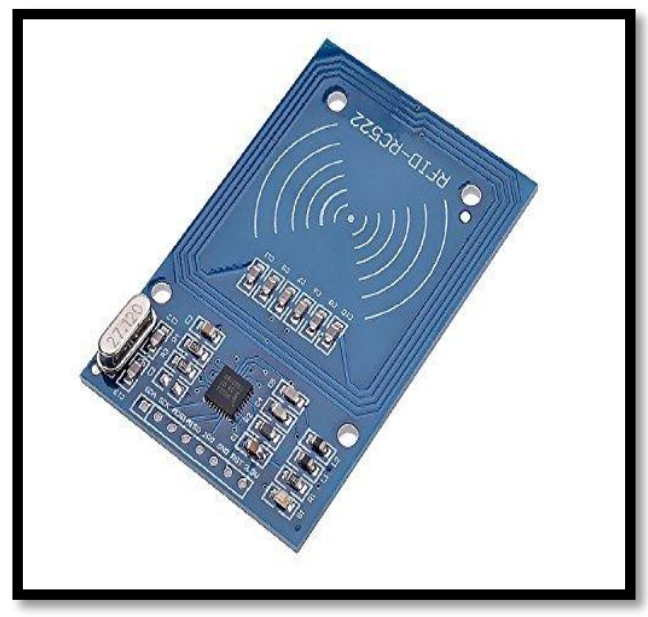

Source:https://robu.in/product/mifare-rfid-readerwriter-1356mhz-rc522-spi-s50-fudan-card-and-keychain/ 
* REES 52: This is component used for the digit combination lock as the backup security for the RFID chip. This ensures that if the RFID fails to work or is breached, this will be the backup to open the door making it a two layer protection.

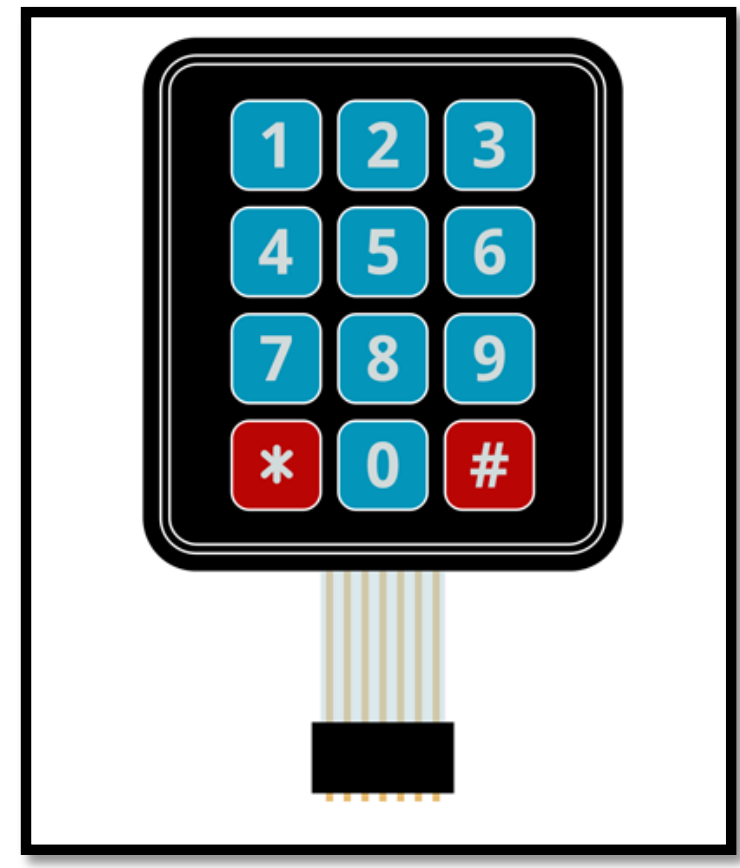

Source: https://www.electroschematics.com/arduino-withkeypad/

* Arduino Uno: Arduino Uno is the circuit board or the control hub of all the components and also the power source for the components. All the data is stored here and processed.

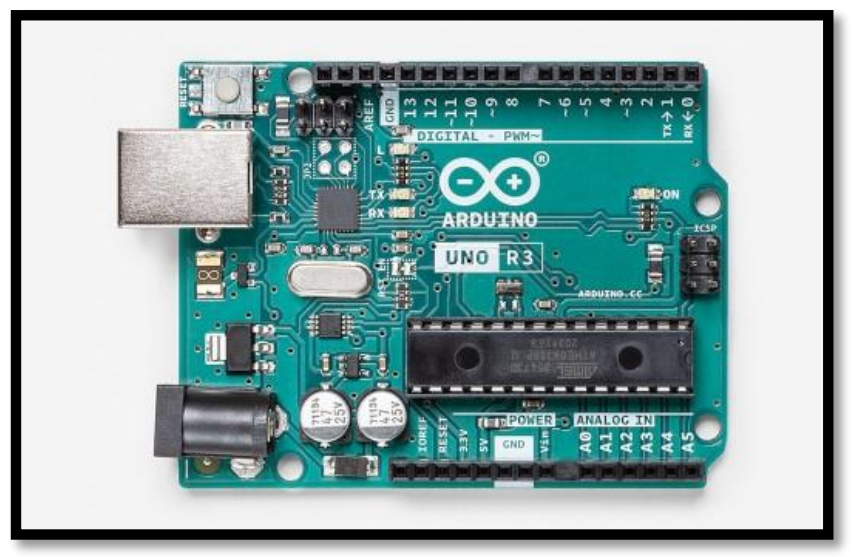

Sourcehttps://store.arduino.cc/usa/arduino-uno-rev3

* Buzzer: This is one of the extra components to improve the aesthetics and working of the security. It helps to know when the entered RFID tag or the number combination is wrong.

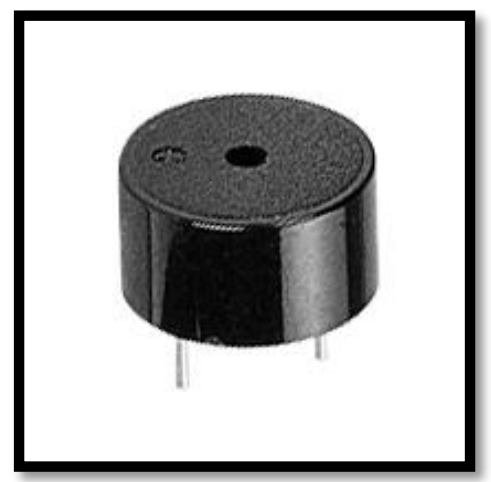

Source: https://shallowsky.com/arduino/class/buzzer.html

\section{Block Diagram:}

A fully functional Arduino security system with all the components mentioned above can be simplified using a block diagram. The RFID reader, REES 52 are connected to Arduino Uno which processes and if correct tag and combination are entered, then the door is unlocked, otherwise the buzzer which is also connected is switched on to tell that either one of them or both of them are wrong.

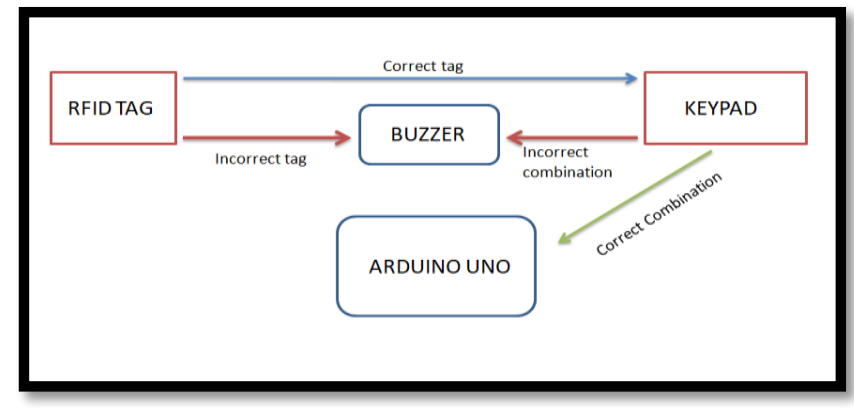

Source: own diagram of author

\section{Concept:}

The RFID tag is placed on the RFID reader and then the reader reads the data from the tag. The correct data will allow the user to proceed with the next step that will be to punch in the number combination in the keypad. The other outcome could be, if the tag is not matched with the data stored in the system then the buzzer will signal by being played once to communicate that the tag is invalid. Also, if the user inputs the wrong combination in the keypad then the buzzer will be signaled and the user has to start from the beginning from the tag reader.

The door will be unlocked for 10 seconds which will be indicated by an LED on the door handle alongside the keypad. If the LED is not on even after the number combination is inserted, that might indicate that there is something wrong with the combination or a very unlikely case, the door. The basic sample codes and schematics are shown in given diagram. 

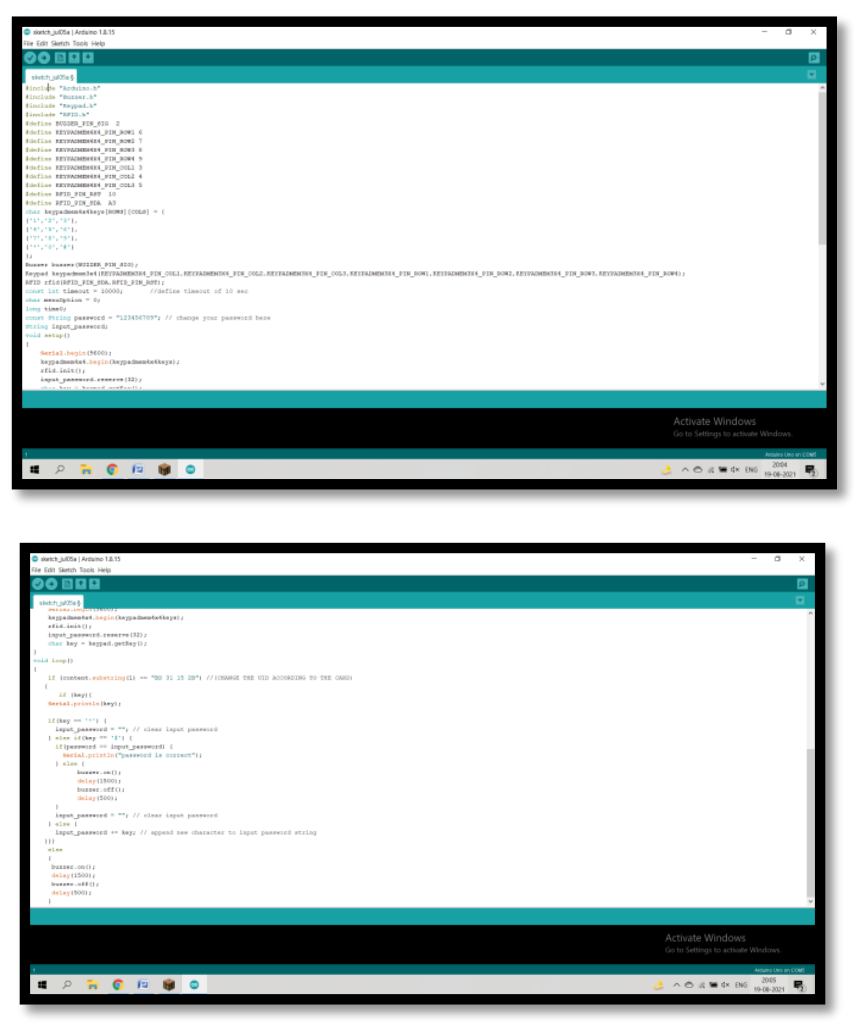

Source: author

\section{Conclusion:}

Arduino based RFID system alongside the numeric combination can be very secure and hassle free. The reason being for this is that RFID system is contact-less and cheap while the numeric combination can be as long as the user wants hence removing the barrier of having only 4 to 8 digit combination which can be brute-forced. This basically means that the whole system is tailor made for the user and also the user can change the password and add tags when necessary by simply using the Arduino software.

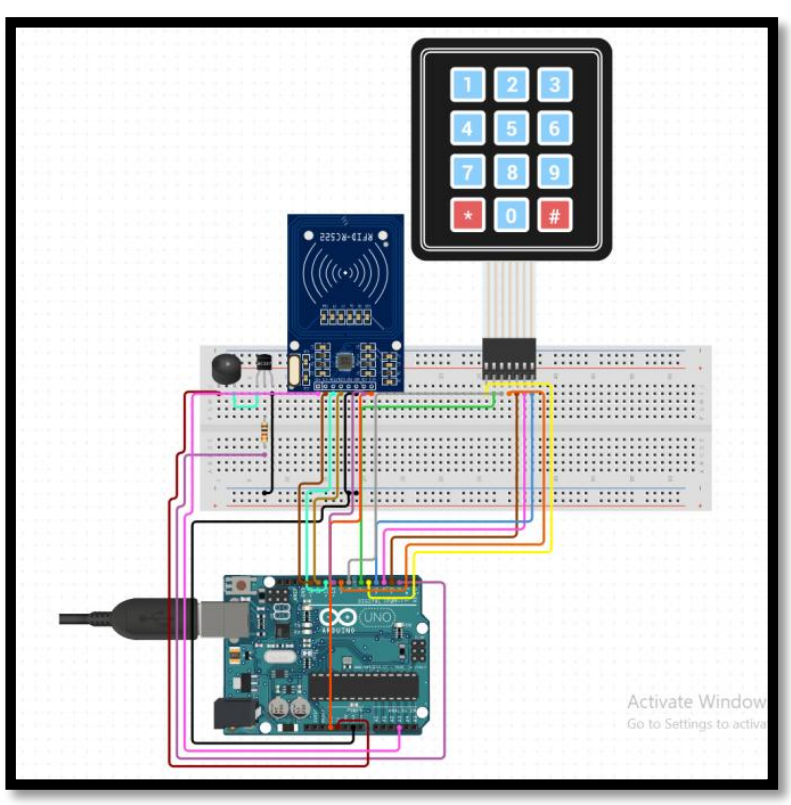

\section{Future Scope:}

The theory of this project is very simple can be modified for whichever purpose deemed necessary. We could also add wifi module to this so that it can connect to the home wifi and send a message if wrong tag or combination is inserted. Also, multiple different tag and numeric combinations can be added, so that each tag has a specific combination implying that each user of the same lock can have their own custom combination. Furthermore, it could be used at different places like door, DIY home safe, etc.

\section{References:}

[1] Retrieved from URL

[2] https://store.arduino.cc/usa/arduino-uno-rev3

[3] Retrieved from URL

[4] https://shallowsky.com/arduino/class/buzzer.html

[5] Retrieved from URL

[6] https://www.mikroe.com/rfid-tag-1356mhz

[7] Retrieved from URL

[8] https://robu.in/product/mifare-rfid-readerwriter-1356mhz-rc522-spi-s50-fudan-card-and-keychain/

[9] Retrieved from URL

[10] https://www.electroschematics.com/arduino-withkeypad/

[11] Retrieved from URL

[12] https://www.circuito.io/app?components $=512,8654,110$ $\underline{21,761981,956215}$

[13] Retrieved from URL

[14] https://create.arduino.cc/projecthub/muhammadaqib/rfid-and-keypad-door-lock-and-alert-systemusing-arduino-60f050 